\title{
BARRERAS PARA LA CALIDAD DE INFORMACIÓN EN ESTABLECIMIENTOS DE SALUD DE LA AMAZONÍA: EL CASO DE TRES SISTEMAS DE INFORMACIÓN DE VIH/SIDA, HEPATITIS B Y SÍFILIS CONGÉNITA
}

\author{
Helen Palma-Pinedoa, Mary Reyes-Vega ${ }^{1, b}$
}

\begin{abstract}
RESUMEN
Objetivos. Identificar las barreras para la calidad de la información en establecimientos de salud de la Amazonía, explorando el caso de tres sistemas de información en salud: la vigilancia epidemiológica, el registro diario de atenciones y registro de defunciones vinculados al $\mathrm{VIH} /$ Sida, hepatitis B y sífilis congénita. Material y métodos. Se aplicó una metodología cuali-cuantitativa. Por un lado, se realizaron 57 entrevistas semi-estructuradas a personal administrativo y asistencial de establecimientos de salud; por otro, se aplicó una encuesta. Participaron 120 establecimientos de salud de las regiones Amazonas, Huánuco, Loreto, Madre de Dios, San Martín y Ucayali. Resultados. Se identificaron ocho barreras vinculadas principalmente al recurso humano, a aspectos socioculturales y a debilidades del propio sistema de salud. Conclusiones. Las barreras identificadas para la calidad de la información en los sistemas de información seleccionados, en establecimientos de salud de la Amazonía, reflejan un problema multidimensional, por lo que estrategias de mejora en el tema deberían ser consideradas.
\end{abstract}

Palabras clave: Sistemas de información; Población indígena; VIH; Hepatitis B; Sífilis (fuente: DeCS BIREME).

\section{BARRIERS FOR THE QUALITY OF INFORMATION IN HEALTH FACILITIES OF AMAZON REGION: THE CASE OF THREE INFORMATION SYSTEMS OF HIV/AIDS, HEPATITIS B, AND CONGENITAL SYPHILIS}

\begin{abstract}
Objectives. To identify the barriers to the quality of information in health facilities in the Amazon region, exploring the case of three health information systems: the epidemiological vigilance, the daily recording of service, and the registry of deaths linked to HIVIAIDS, hepatitis B, and congenital syphilis. Materials and Methods. A quali-quantitative methodology was applied. On the one hand, 57 semi-structured interviews were conducted with administrative and assistance staff at health facilities; on the other hand, a survey was applied. One hundred and twenty health facilities in the regions of Amazonas, Huánuco, Loreto, Madre de Dios, San Martín, and Ucayali. Results. Eight barriers were identified, which were linked mainly to the human resource, to sociocultural aspects, and weaknesses of the health system. Conclusions. The barriers identified for the quality of the information in the selected information systems in health facilities in the Amazon region reflect a multidimensional problem, so strategies for improvement in the subject should be considered.
\end{abstract}

Keywords: Information systems; Indigenous population; HIV; Hepatitis B; Syphilis (source: MeSH NLM).

\section{INTRODUCCIÓN}

Un sistema de información en salud (SIS) se define como un mecanismo de recolección, procesamiento, análisis y transmisión de información necesaria para organizar y operar los servicios de salud, así como para la investigación y gestión con vistas al control de las enfermedades. La información producto de este sistema representa un bien público por su trascendencia para el diseño, seguimiento y evaluación de las políticas públicas, además de permitir evaluar los cambios a nivel del estado de la salud, del sistema de salud y sus determinantes. No obstante, su utilidad puede verse reducida debido a limitaciones en la calidad de los datos, su análisis, uso y difusión ${ }^{(1-3)}$.

\footnotetext{
Centro Nacional de Epidemiología, Prevención y Control de Enfermedades. Ministerio de Salud. Lima, Perú.

Antropóloga, Magíster en Salud Pública; ${ }^{\mathrm{b}}$ Médico, Magíster en Epidemiología.

Recibido: 14/03/2017 Aprobado: 31/01/2018 En línea: 05/04/2018
}

Citar como: Palma-Pinedo H, Reyes-Vega M. Barreras para la calidad de información en establecimientos de salud de la Amazonía: El caso de tres sistemas de información de VIH/Sida, hepatitis B y sífilis congénita. Perú. Rev Peru Med Exp Salud Publica. 2018;35(1):25-31. doi: 10.17843/rpmesp.2018.351.3596. 
La calidad de los procesos y productos vinculados a los SIS abarca aspectos como la consistencia, correspondencia y otros criterios que permiten que los datos obtenidos reflejen la realidad tal como es. En este marco, la calidad de la información resulta vital para el desarrollo de la inteligencia sanitaria, ya que si los datos no son veraces ni completos la información que se producirá no permitirá tomar decisiones efectivas ni eficientes ${ }^{(4-6)}$.

Las zonas rurales de países en vías de desarrollo se encuentran marcadas por la pobreza, desigualdad económica y el acceso limitado a bienes y servicios. La geografía accidentada, la insuficiente inversión en infraestructura, recursos humanos y equipamiento tienen efecto en los SIS que al no registrar datos o al no hacerlo con calidad, impiden evidenciar los problemas de salud de ciertos sectores de la población, como la población indígena amazónica. Es sabido también que los datos provenientes del primer nivel de atención presentan una calidad heterogénea, algo que se suele observar con claridad en el nivel central al consolidar esta información. Dicha heterogeneidad refleja la diversidad de las realidades locales que presentan diferentes condiciones socioeconómicas, capacidades organizativas, capacidades administrativas y de prestación de servicios, los cuales afectan la calidad de la información generada ${ }^{(7-10)}$.

En el Perú, los SIS que proveen insumos para la elaboración de los perfiles epidemiológicos así como indicadores para el monitoreo y evaluación de las intervenciones orientadas a los problemas de salud pública son responsabilidad principalmente del Ministerio de Salud (MINSA) ${ }^{(11-13)}$. Para el caso del $\mathrm{VIH}$, hepatitis $\mathrm{B}$ y sífilis, las cuales son frecuentes en población de comunidades indígenas amazónicas ${ }^{(14-18)}$, los principales SIS son la vigilancia epidemiológica a cargo del Centro Nacional de Epidemiología, Prevención y Control de Enfermedades; así como el registro diario de atenciones (HIS) y el registro de defunciones a cargo de la Oficina General de Tecnologías de la Información (13,19-21).

El objetivo del presente estudio fue identificar las barreras para alcanzar la calidad de la información en establecimientos de salud que atienden a población indígena amazónica a partir del caso de tres SIS (vigilancia epidemiológica, HIS y registro de defunciones) de VIH/ Sida, hepatitis $B$ y sífilis congénita.

\section{MATERIALES Y MÉTODOS}

\section{DISEÑO DEL ESTUDIO}

La presente investigación empleó una metodología mixta, es decir, que presenta un componente cualitativo (estudio de caso) el cual se complementa con un componente cuantitativo (estudio transversal). Una de las principales ventajas de este diseño es que permite tener una perspectiva holística del fenómeno investigado: frecuencia, amplitud y magnitud (cuantitativa), así como profundidad

\section{MENSAJES CLAVE}

Motivación para realizar el estudio. Se ha descrito en otros países que la geografía accidentada, la insuficiente inversión en infraestructura, recursos humanos y equipamiento tienen efecto en los sistemas de información en salud (SIS), los cuales al no registrar datos o al no hacerlo con calidad impiden evidenciar los problemas de salud de ciertos sectores de la población, como las poblaciones indígenas. Estos aspectos no han sido estudiados previamente en el Perú. Este estudio tuvo por objetivo identificar las barreras para alcanzar la calidad de la información en establecimientos de salud que atienden a población indígena amazónica a partir de tres SIS (vigilancia epidemiológica, HIS y registro de defunciones), considerando la información relacionada a VIH/Sida, hepatitis B y síflis congénita.

Principales hallazgos. Se identificaron barreras vinculadas al recurso humano, a la falta de priorización de este tema en la institución, la fragmentación y competencia entre los SIS, la falta de presupuesto, barreras socioculturales y barreras relacionadas a búsqueda de casos, tamizaje y calidad de atención.

Implicancias. Estas barreras identifica para la calidad de la información en establecimientos de la amazonía reflejan un problema multidimensional, por lo que se deben establecer estrategias que permitan aminorarlas y de esa manera mejorar la calidad de la información procedente de la atención a la población indígena amazónica, lo cual apoyará la toma de decisiones en salud pública a nivel regional y nacional.

y complejidad (cualitativa); además de generalización (cuantitativa) y comprensión (cualitativa) ${ }^{(22)}$.

El estudio fue propuesto por el Centro Nacional de Epidemiología, Prevención y Control de Enfermedades del MINSA, y se desarrolló en seis regiones: Amazonas, Huánuco, Loreto, Madre de Dios, San Martín y Ucayali. La recolección de la información se realizó durante el período setiembre-diciembre 2014. Con la finalidad de definir los ámbitos a trabajar dentro de cada región, se establecieron dos criterios de inclusión:

- Establecimientos de Salud (EE.SS.) que hayan notificado casos de VIH y/o Sida en el periodo 20102014, según sus respectivos subsistemas de vigilancia epidemiológica.

- EE. SS. del MINSA que atendían a población indígena amazónica según el II Censo de Comunidades Indígenas de la amazonía peruana de 2007.

A partir de ello, se encontró que 122 establecimientos cumplieron con estos criterios. Esta información fue validada posteriormente por el personal de las direcciones regionales de salud participantes del estudio.

\section{COMPONENTE CUALITATIVO}

Los datos cualitativos se construyeron a partir de entrevistas semi-estructuradas aplicadas de forma individual y grupal. La guía de entrevista, validada mediante juicio de expertos, contenía 22 preguntas que fueron realizadas a todos los informantes, y que indagaban en cinco aspectos: (i) características del informante y el establecimiento de salud; (ii) barreras identificadas para 
el registro, (iii) barreras identificadas para el acopio, flujo de información, procesamiento y control de calidad; (iv) barreras identificadas para la retroalimentación, difusión de la información; (v) propuestas de mejora.

Se entrevistó a 54 trabajadores pertenecientes a los 122 establecimientos seleccionados, así como a tres funcionarios del nivel central (57 en total), a partir de 39 entrevistas individuales y tres grupales. De los 54,11 pertenecían a alguna Diresa, 15 a una Red de salud, 18 a una microrred, y 10 a un puesto de salud. Esto permitió conocer el desarrollo de los SIS y sus flujos, y fue además un mecanismo de validez que permitió contrastar la información. El 84\% fue personal profesional, mientras que el resto era técnico. El número de entrevistados se determinó por la accesibilidad presente en cada visita de campo. El criterio de selección fue ser responsable de alguna de las siguientes áreas: Epidemiología; Estadística; Estrategia para la Prevención y Control de ITS, VIH y Sida; y Laboratorio de los EE. SS. seleccionados. Esto, por ser las que se vinculan directamente con el tema de interés del estudio. Evidentemente, en los niveles inferiores, sobre todo en los puestos de salud, una misma persona tenía a cargo más de una o todas las áreas mencionadas.

Las entrevistas fueron realizadas en las horas y lugares elegidos por los informantes para su comodidad, y fueron aplicadas por la antropóloga miembro del equipo de investigación, las cuales en todos los casos no tuvieron una duración menor a los 30 minutos. El consentimiento informado obtenido fue verbal, y fue solicitado antes de dar inicio a las entrevistas. Además, estas fueron grabadas, transcritas, y analizadas siguiendo un análisis temático a partir de la codificación lo que permitió la identificación de patrones, así como la relación entre conceptos y procesos descritos por los entrevistados. La codificación fue realizada con el software Atlas.ti 7.0.

\section{COMPONENTE CUANTITATIVO}

De un total de 122 EE. SS. que cumplieron los criterios de inclusión, participaron 120 ya que dos no retornaron las encuestas por lejanía geográfica (Tabla 1).
Se elaboraron dos cuestionarios, una para ser aplicada a nivel del EE. SS. u hospital seleccionado; y la otra para ser aplicada en la microrred/red/Diresa a la cual pertenecía dicho EE. SS. Los instrumentos permitieron la recolección de datos referente a la vigilancia epidemiológica, HIS, certificados de defunción y búsqueda/detección de casos de infecciones por $\mathrm{VIH}$, hepatitis $\mathrm{B}$ y sífilis. Los cuestionarios fueron validados mediante apreciación de juicio de expertos (6 en total) en una reunión con representantes de cada una de las regiones incluidas, lo que permitió ajustar el instrumento a la realidad presente en dichos contextos.

La recolección de datos se realizó mediante encuesta con cuestionario autoaplicado para lo cual se contó con el apoyo de coordinadores de estudio en cada región seleccionada, quienes fueron previamente capacitados en el protocolo del estudio, el llenado de los cuestionarios y en su monitoreo. Además se realizó una visita de supervisión en cada región para vigilar el proceso a cargo de las responsables del estudio. Los datos fueron recolectados a partir de personal de salud encargado del registro en los niveles asistenciales y profesionales de los niveles administrativos responsables de consolidar, hacer el control de calidad, y analizar los datos. Al igual que en el caso cualitativo, se priorizó al personal de epidemiología y estadística. El personal que realizó el llenado de los cuestionarios validó la información proporcionada mediante su firma y sello. El análisis estadístico fue realizado con el programa SPSS versión 23.0 para Windows. Se realizó estadística univariada basada en la obtención de frecuencias y porcentajes.

Los informantes aceptaron voluntariamente serencuestados o entrevistados, y previamente fueron informados sobre los objetivos del estudio. Los cuestionarios fueron codificados para mantener la confidencialidad respecto a la identidad de los informantes. La investigación fue aprobada por el Comité de Ética del Instituto de Medicina Tropical "Daniel Alcides Carrión" de la Universidad Nacional Mayor de San Marcos.

Tabla 1. Establecimientos de salud participantes según dirección regional de salud

\begin{tabular}{lcccc}
\hline & \multicolumn{3}{c}{ Número de establecimientos } & \multirow{2}{*}{ Total } \\
\cline { 2 - 5 } Regiones & Puestos de salud & Centros de salud & Hospitales & 4 \\
\hline Amazonas & 22 & 10 & 1 & 36 \\
Huánuco & 3 & 3 & 3 & 7 \\
Loreto & 12 & 18 & 2 & 33 \\
Madre de Dios & 3 & 6 & 12 & 11 \\
San Martín & 7 & 8 & 2 & 27 \\
Ucayali & 0 & 4 & & 6 \\
\hline
\end{tabular}




\section{RESULTADOS}

A partir de las entrevistas y encuestas realizadas se identificaron distintas barreras, algunas que afectan la calidad de los datos de los SIS directamente, y otras que lo hacen indirectamente:

\section{BARRERAS VINCULADAS AL RECURSO HUMANO}

Capacitación insuficiente: se mencionó que este problema nace desde la ausencia de los SIS y relevancia de la calidad de los datos como temática en la formación de los profesionales de la salud, que al iniciarse en la atención se encuentran con la labor administrativa que deben realizar además de la asistencial. Esto intenta subsanarse con capacitaciones desde el nivel central hacia las direcciones regionales, que van perdiendo fuerza cuando llegan a las redes y luego microrredes.

«El personal de salud que llena HIS necesita una capacitación, nosotros ponemos toda nuestra parte para estar en línea con ellos porque nosotros tampoco hemos sido capacitados, solo nos guiamos por internet, por los manuales. Sería importante que haya un personal que nos explique porque nosotros también tenemos dudas (Personal de estadística - Red Datem del Marañón - Loreto)».

Al respecto, la encuesta evidenció que en el caso de la vigilancia epidemiológica, un alto porcentaje de los responsables del llenado de la ficha de notificación de caso de $\mathrm{VIH}(35,8 \%)$, hepatitis $\mathrm{B}(71,7 \%)$ y sifilis congénita $(71,7 \%)$ nunca habían recibido capacitación para este fin. EI $55,8 \%$ de los EE. SS. reportaron que su personal nunca fue capacitado para el llenado del certificado de defunción, así como el $17,5 \%$ en el caso del registro del HIS.

La principal consecuencia de la falta de capacitación es el mal llenado. Un ejemplo es que en el caso del registro de defunciones, se observó alto grado de dificultad para completar el certificado de defunción por VIH (39\%), hepatitis $B(38 \%)$ y sífilis congénita (30\%). En dichos EE. SS. siempre o casi siempre se dejaba sin llenar el código CIE-10 cuando se trataba de una defunción por $\mathrm{VIH}$ $(30,5 \%)$, hepatitis $B(62,5 \%)$ y sífilis $(20 \%)$.

Escasez de recursos humanos: los entrevistados señalaron que la falta de recursos humanos genera consecuencias en los SIS, lo que se observa en un dato mal generado a partir de la sobrecarga de las tareas del personal que debe asumir funciones adicionales al registro de datos, siendo los EE. SS. del primer nivel de atención los más afectados.

Para el caso del HIS, esta barrera ha contribuido a la formación de una mala práctica que es no realizar su llenado al culminar la atención o al final de día, sino posponerlo para el fin de semana o mes favoreciendo la mala calidad del dato. Dado que el llenado se hace a contratiempo se propicia: la mala codificación, datos incompletos, letra poco legible, entre otros.
"Los FUA (Formato único de Atención) y las fichas las llenamos en el momento, el HIS es el problema. Se supone que luego que el paciente es atendido debería ser llenado todo, pero como la demanda es alta lo dejamos para después". (Responsable del C.S Iñapari - Microrred lberia - Madre de Dios).

Rotación del personal: se señaló que la rotación del personal dificulta la transferencia de información y/o tecnología, lo que hace difícil contar con un recurso con preparación y experiencia; repercutiendo en este caso en el correcto desarrollo de los SIS y en la calidad de sus datos. En la amazonía, especialmente en las zonas rurales, el problema de la rotación se agudiza. Las condiciones de vida y de trabajo (sobre todo en las comunidades indígenas) hacen que el personal permanezca poco tiempo.

«Un problema es que el personal es muy volátil, la capacitación se pierde, hace que lo que se había logrado se pierda. Tratamos que el personal sea de planta, pero eso lo podemos hacer más en Lima que en las regiones, y ese es un problema para todas las transferencias tecnológicas». (Responsable de la red nacional de laboratorios de ITS y VIH/Sida del Instituto Nacional de Salud)

Falta de recursos para la captación y tamizaje: los entrevistados señalaron que existían limitaciones a causa de la falta de recursos, lo que hacía que las campañas de tamizaje se desarrollen principalmente en las ciudades, dejando de lado otros espacios como los ámbitos de residencia de la población indígena. Este es un factor que tiene un efecto secundario en la calidad de los datos de los SIS explorados, al generar subregistro.

«Hasta el año 2009 se tamizaba solo a las mujeres, sin embargo, desde el 2010 se tamiza a los varones y es ahí cuando se encuentran más casos. Eso antes no aparecía en los registros. Es curioso, pero felizmente se retomó y eso nos permitió encontrar más casos. Pero si nosotros esperamos a los varones en los establecimientos de salud, no acuden; por eso siempre tratamos de ir a las universidades e institutos a hacer los tamizajes $y$ ahi es donde encontramos. Antes tampoco se trabajaba en los campos mineros pero ahora sí se hace». (Responsable de la Estrategia de VIH/Sida, hepatitis B y Sífilis - Diresa Madre de Dios)

Escasez de recursos para el diagnóstico: se manifestó que la falta de estos recursos impide confirmar los casos y hacerlos visibles en los SIS. Un ejemplo claro es lo que sucede para el diagnóstico de hepatitis B, ya que existía carencia del material necesario para el diagnóstico así como de personal entrenado para hacerlo. Por ello se observa que el número de casos registrados era bajo a pesar de ser conocida su alta prevalencia en la amazonía.

«E: ¿En lo que es hepatitis B se hacen tamizajes?

No, no contamos con pruebas.

E: ¿Eso es de ahora o viene desde antes?

Nos adecuamos, pero siempre nos falta material, los reactivos tenemos con las justas para las gestantes pero no para el resto de la población. Recién ahora nos enviaron para sifilis y con eso hemos salido». (Responsable del P.S San Juan Grande-Microrred Colorado-Madre de Dios). 
Así, la encuesta permitió evidenciar que alrededor del $76 \%$ de los EE. SS. notificaron de forma continua casos de $\mathrm{VIH} /$ Sida y sífilis congénita en comparación con el porcentaje (54\%) que lo hicieron para hepatitis B. Se observó que para el caso de hepatitis $B$, el número bajo de casos notificados podría obedecer a subregistro por no contar con los insumos para el diagnóstico, pues el $31,3 \%$ de los EE. SS. que realizan pruebas para hepatitis $B$, refirieron que "siempre" o "casi siempre" se encuentran desabastecidos de pruebas.

\section{Ausencia de mecanismos cotidianos para asegurar la fiabilidad de los datos: se mencionó la falta de mecanismos aplicados cotidianamente que aseguren la fiabilidad de los datos, principalmente por la falta de recursos humanos y la sobrecarga laboral. Esto partiría de que este tema queda relegado frente a otras prioridades, sobre todo en los EE. SS. que realizan labor asistencial.}

«E: ¿Qué mecanismos tienen para el control de calidad de sus datos?

Lo estábamos haciendo pero hace ya como medio año que lo hemos dejado.

\section{E: ¿Cómo lo hacían?}

Control de calidad hay en varios aspectos, de las muestras de laboratorio, de las historias clínicas, de cómo va la atención al usuario. Antes lo hacíamos con la revisión o supervisión de las historias clínicas, pero ya no lo hacemos.

\section{E: ¿Por qué?}

De repente se han debilitado las comisiones, antes lo hacía un equipo». (Responsable de estrategia de $\mathrm{VIH} / \mathrm{Sida}$, hepatitis $\mathrm{B}$ y Sífilis del C.S Laberinto - Microrred Laberinto - Madre de Dios)

La encuesta evidenció que solo el $55 \%$ de EE. SS. que presentaron casos de infección por $\mathrm{VIH}$, hepatitis $\mathrm{B}$ o sífilis congénita, realizaron control de calidad "siempre" o "casi siempre" a las fichas de notificación cruzando la información con otros registros disponibles en el establecimiento. En el caso de las hojas HIS, en $48,7 \%$ de los EE. SS. el personal que registró realizó control de calidad "siempre" o "casi siempre", y en el 58,4\% lo realizó el personal de estadística cuando estos fueron ya recepcionados. En el caso de los certificados de defunción, el control de calidad por el personal que registra solo se realizó en el $30 \%$ de establecimientos y el control de calidad por el personal de estadística en el $34 \%$.

Fragmentación y competencia entre los distintos SIS: se observó que ante la ausencia de un sistema único integrado de información existen sistemas que responden a distintos intereses y objetivos. Esta falta de unidad genera competencia entre sistemas más posicionados en los que el personal invierte más esfuerzo en detrimento del resto. Un ejemplo es el correspondiente al Seguro Integral de Salud que adquirió preponderancia por ser una prioridad para la gestión desde el nivel central y porque su no llenado conllevaba una pérdida económica o falta de reconocimiento del pago por los servicios de atención; siendo el HIS el principal perjudicado.

"Antes se daba mayor énfasis a lo que es el Seguro Integral de Salud porque es lo que les generaba ingresos en el primer nivel, y por eso al HIS lo dejaban de lado. Para ellos no les producía, no les servía y lo dejaban de lado. Pero como ahora se ha empezado con lo que es presupuesto por resultados ya se está poniendo más énfasis al HIS». (Responsable de Estadística - Red Alto Amazonas - Loreto)

\section{BARRERAS VINCULADAS A DEBILIDADES DEL PROPIO SISTEMA DE SALUD}

Falta de presupuesto: se indicó que a nivel regional hay una falta de inversión en los SIS, lo que hace que estos cuenten con un magro presupuesto para su operatividad. Un discurso recurrente que se recogió durante las entrevistas fue que las oficinas de estadística, al igual que las de epidemiología, no manejan un presupuesto propio y no participan del presupuesto por resultados. Sus actividades y demás gastos estaban supeditados a la voluntad de las otras oficinas y sobre todo de las estrategias sanitarias.

"Acá en la red de salud ejecutora no tenemos presupuesto, dependemos directamente de la administración o la dirección y eso es una limitante porque necesitamos salir, monitorear los puntos de digitación, pero no tenemos la logística para hacerlo, es muy burocrático». (Responsable de estadística- Red Alto Amazonas-Loreto)

\section{BARRERAS SOCIOCULTURALES}

se identificó que existen barreras socioculturales que presentan una afectación secundaria en la calidad de los datos de los SIS explorados, en la medida que estas generan subregistro. Por ejemplo, se mencionó que el estigma y discriminación afecta principalmente a los pacientes con $\mathrm{VIH}$ y conlleva a que estos prefieran no acudir a un EE.SS. o den información falsa. Además, en algunos grupos indígenas presentes en las regiones estudiadas se mencionó que existía un fuerte estigma que llevaba a la expulsión de aquellos que se presumía tenían VIH, lo que se apreció en el grupo awajún presente en Amazonas y Loreto.

"Las barreras culturales son extremadamente marcadas. Por ejemplo ayer escuchaba que un dirigente nativo decía 'acá todo se sabe, entre nosotros no hay secretos, en el pueblo indígena no hay secretos'. Primero que hay una ley de confidencialidad, pero en el caso de ellos es el Apu el que decide. Si uno va y hace una campaña de salud el Apu quiere ver los resultados (...) Entonces si existe la ley contra el Sida ellos dirán 'existe pero para los peruanos, no para nosotros'. Entonces eso nos genera problemas, porque convencerlos es difícil, y después que estén señalando quién tiene Sida y quién no, porque incluso en algunos casos los botan de la comunidad. Eso es algo bastante traumático, imagínese el hecho de darle los resultados al Apu, estaríamos yendo en contra de la ética y lo que dice la norma»(Responsable de epidemiología - Diresa Amazonas)

Por otro lado, se señaló que los síntomas vinculados al VIH/ Sida eran reconocidos como "daño" o brujería en varios de los pueblos indígenas, por lo cual las personas llamadas a restablecer la salud no eran las de los EE.SS. sino los curanderos y otros especialistas de la medicina tradicional, y por ello, estos casos no eran registrados. 
«En un caso de VIH ellos no pensaban que era eso, pensaban que era daño, un embrujo, por eso se iban por otras opciones. Pero la información sobre de qué se trata la enfermedad, cómo se contagia (transmite) le hemos dado pero ellos no toman consciencia en que eso les puede dar». (Responsable del C.S Yuyapichis- Microrred Puerto Inca-Huánuco)

\section{DISCUSIÓN}

Los SIS constituyen herramientas para el monitoreo y evaluación de intervenciones preventivas, de diagnóstico temprano y tratamiento de las enfermedades así como del acceso a servicios de salud ${ }^{(23-25)}$. También han mostrado ser efectivos en procesos para la mejora de la calidad de atención, reducción del uso innecesario de estudios de laboratorio, reducción de costos, detección y prevención de reacciones adversas medicamentos, logística, etc ${ }^{25)}$.

Sin embargo, la falta de calidad en los SIS y la información resultante no es un problema nuevo en el Perú y en la región de las Américas. El presente estudio muestra que las barreras para alcanzar la calidad de los datos en establecimientos de salud de la Amazonía son múltiples.

Los problemas alrededor de la calidad de los datos, aunque en distinta medida, se hacen presentes en todos los niveles analizados, desde el nivel nacional, nivel regional, hospitales y el primer nivel de atención. Pero es en aquellos donde se construye el dato y donde se empieza su consolidación donde existen más limitaciones por capacitación insuficiente, escasez y elevada rotación de personal, y donde la carga laboral y administrativa es mayor. Un ejemplo de esto es la preocupante frecuencia de personal que realizaba el llenado de la ficha de notificación de caso de hepatitis B, VIH, HIS y certificado de defunción que nunca fueron capacitados para este fin.

Esto coincide con una investigación cualitativa ${ }^{(26)}$ realizada con funcionarios y trabajadores de EE. SS. públicos y privados de Bostwana, tanto para el nivel nacional, distrital y comunitario, que tuvo como objetivo identificar las fortalezas y debilidades para el manejo de datos en salud y sistemas de reporte usados rutinariamente para monitoreo y evaluación. En dicho estudio los entrevistados reconocieron la necesidad de entrenamiento tanto para el manejo de herramientas para la recolección de datos como para su análisis; asimismo, consideraban necesario contar con personal en número suficiente para la realización de estas actividades con calidad.

Los resultados obtenidos en el presente estudio también coinciden con lo obtenido por Soto-Cáceres ${ }^{(27)}$ respecto de la calidad del llenado de los certificados de defunción emitidos en un hospital público (Chiclayo-Perú) para el período entre julio-setiembre 2006, concluyendo en la necesidad de implementar estrategias de capacitación orientadas a los médicos. A una conclusión similar se llega en un estudio que analiza la calidad de la certificación de las defunciones en Ica demostrando una elevada frecuencia de errores lo que afecta el análisis de la situación de la salud (28).

Un plan de mejora de la calidad de los datos debería considerar todos los eslabones que intervienen en su desarrollo. Uno de los principales puntos debería ser el fortalecimiento de la capacitación del personal. La inversión en este tema es relevante y tiene una elevada relación costo-beneficio; sin embargo, esta no es percibida por los tomadores de decisiones por lo que se priorizan las actividades de atención de salud cuyos beneficios son más evidentes e inmediatos ${ }^{(29)}$. Este problema se observa en la evaluación del sistema de información rutinaria de la Dirección de Salud $\mathrm{V}$ Lima Ciudad, que encontró que el $80 \%$ de las unidades de estadística de los establecimientos de salud de dicho ámbito no contaban con financiamiento específico para sus sistemas de información (11).

Por otro lado, se encontró que la fragmentación de los sistemas de información tiene un impacto en la calidad de los datos. Lo que coincide con lo encontrado por Reyes y Alarcón ${ }^{(30)}$ quienes identifican a la presencia de vías paralelas de flujo de información, así como la falta de procesos estandarizados para el control de calidad, como puntos críticos para el registro.

Una fortaleza del presente estudio es que ha sido realizado con una metodología mixta que se enfocó en la calidad de la información de los tres SIS (HIS, vigilancia epidemiológica y registro de defunciones) como un proceso multidimensional y no se limitó solo al análisis de la problemática del registro de la información que constituye solo una arista del problema, ni solo al aspecto tecnológico. La principal limitación encontrada es el escaso número de investigaciones publicadas acerca de este tema en el Perú y con este enfoque metodológico, lo que ha limitado la comparación de los hallazgos de la presente investigación.

Se concluye que las barreras identificadas para la calidad de la información en establecimientos de la amazonía reflejan un problema multidimensional. Estas fueron las vinculadas principalmente al recurso humano, aspectos socioculturales y a debilidades del propio sistema de salud. Una de las barreras encontradas y que coinciden con estudios antes realizados, es la falta de capacitación del personal responsable del registro, por lo que se deben establecer estrategias que permitan disminuir esta y otras barreras para de esa manera mejorar la calidad de la información procedente de establecimientos que atienden a población indígena amazónica.

Contribuciones de autoría: HPP y MRV han participado en la concepción del artículo, la recolección y análisis de los datos, redacción del artículo y aprobación de la versión final.

Fuentes de financiamiento: Centro Nacional de Epidemiología, Prevención y Control de Enfermedades.

Conflictos de interés: Los autores declaran no tener conflictos de interés. 


\section{REFERENCIAS BIBLIOGRÁFICAS}

1. Organización Mundial de la Salud. Informe sobre la Salud en el Mundo. Génova: WHO; 2003.

2. Aqil A, Lippeveld T, Hozumi D. PRISM framework: a paradigm shift for designing, strengthening and evaluating routine health information systems. Health Policy Plan. 2009;24(3):217-28.

3. Alazraqui M, Mota E, Spinelli. Sistemas de Información en Salud: de sistemas cerrados a la ciudadanía social. Un desafío en la reducción de desigualdades en la gestión local. Cad. Saúde Pública. 2006;22(12):2693-2702.

4. Aqil A, Orabaton N, Azim T, Hiroshi A, Hozumi D, Lippeveld T. Determinants of Performance of Routine Health Information System (RHIS): Evidence from Uganda and Pakistan. American Public Health Association 133rd Annual Meeting \& Exposition. Philadelphia, 2005

5. Chae YM, Kim SI, LeeBH, ChoiSH, Kim IS. Implementing health management information systems: measuring success in Korea's health centers. Int J Health Plann Manage 1994;9(4):341-8.

6. Da Silva AS, Laprega MR. Critical evaluation of the primary care information system (SIAB) and its implementation in Ribeiero Preto, Sau Paulo, Brazil. Cad Saude Publica 2005;21(6):1821-8.

7. Alazraqui M, Mota E, Spinelli . Sistemas de Información en Salud: de sistemas cerrados a la ciudadanía social. Un desafío en la reducción de desigualdades en la gestión local. Cad. Saúde Pública. 2006;22(12):2693-702.

8. Health ND: The EPI Situation in Mozambique: Ministry of Health. Mozambique; 2002.

9. Mavimbe JC, Braa J, Bjune G. Assessing immunization data quality from routine reports in Mozambique. BMC Public Health. 2005; 5:108.

10. Odhiambo-Otieno GW, Odero WW. Evaluation criteria for the district health management information systems: lessons from the Ministry of Health, Kenya. Afr Health Sci. 2005;5(1):59-64.

11. Vargas J, Reyes N, Rantes H, Aquino $\mathrm{S}$, Asca J. Evaluación del Sistema de Información Rutinaria de la Dirección de Salud V Lima Ciudad. Rev Peru Epidemiol. 2010;14(1) [p. 7].

12. Ministerio de Salud del Perú. Evaluación del Sistema de Información en Salud del Perú. Lima: MINSA; 2008.
13. Estrategia Sanitaria Nacional de Prevención y Control de Infecciones de Transmisión Sexual VIH/Sida. Manual de registro y codificación de la atención en la consulta externa. Lima: MINSA; 2012.

14. Zavaleta C, Fernández C, Konda K, Valderrama Y,VermundS, Gotuzzo E. High prevalence oh HIV and syphilis in a remote native community of the peruvian amazon. Am J Trop Med Hyg, 2007;76(4):703-5.

15. Ormaeche M, Whittembury A, Pun M, Suarez-Ognio L. Hepatitis B virus, syphilis, and HIV seroprevalence in pregnant women and their male partners from six indigenous populations of the Peruvian Amazon Basin. Int J Infect Dis. 2012;16(10):e724-30. doi: 10.1016/j. ijid.2012.05.1032

I6. Indacochea $S$, Gotuzzo E, De la Fuente J, Phillips I, Whignal S. Elevada prevalencia de marcadores de hepatitis B y Delta en el valle interandino de Abancay. Rev Med Hered. 1991;2(4):168-72.

17. Bartletta E, Zavaleta C, Fernández C, Razuri H, Vilcaromero S, Vermunsa $S$, et al. Expansion of HIV and syphilis into the Peruvian Amazon: a survey of four communities of an indigenous Amazonian ethnic group. Int J Infect Dis 2008;12(6): 89-94.

18. Alva I, Orellana R. Social and contextual factors that influence HIV risk behaviors among indigenous MSM in the Peruvian Amazon. Retrovirol 2012;9(Suppl):112.

19. Dirección General de Epidemiología. NTS $\mathrm{N}^{\circ}$ 115- MINSA/DGE V.01 "Norma Técnica de Salud para la Vigilancia Epidemiológica en Salud Pública de la Infección por el Virus de la Inmunodeficiencia Humana (VIH) y de las Infecciones de Transmisión Sexual (ITS) en el Perú. Lima: DGE/MINSA; 2015.

20. Dirección General de Epidemiología. Directiva Sanitaria $\mathrm{N}^{\circ}$ 062-MINSA/ DGE-V.01. Directiva Sanitaria para la Vigilancia Epidemiológica de Sífilis Materna y Síflis Congénita. Lima: DGE/ MINSA; 2015.

21. OficinaGeneraldeEstadísticae Informática. Directiva Administrativa N ${ }^{\circ}$ 166-MINSA/ OGEI-V.01 que establece el Procedimiento para el Flujo y Calidad de los Formularios de Hechos Vitales del Nacido Vivo y de Defunción. Lima: OGEI/MINSA; 2015.

22. Hernández R. Metodología de Investigación. México, D.F: McGraw-Hill Interamericana; 2003.
23. Health Informations System Knowledge Hub, The University of Queensland, University of Washington; Institute for Health Metrics and Evaluation. Improving the quality and use of health information systems: essential strategic issues. Australia, University of Queensland; 2009.

24. Chan M, Kazatchkine M, Lob-Levyt J, Obaid T, Schweizer J, Sidibe M, et al. Meeting the demand for results and accountability: a call for action on health data from eight global health agencies. PLoS Med. 2010;7(1):e1000223.

25. Bates DW, Pappius EM, Kuperman GJ, Sittig D, Burstin H, Fairchild D, et al. Measuring and improving quality using information systems. Stud Health Technol Inform. 1998;52:814-8.

26. Ledikwe JH, Grignon J, Lebelonyane R, Ludick S, Matshediso E, Sento BW, et al. Improving the quality of health information: a qualitative assessment of data management and reporting systems in Botswana. Health Res Policy Syst. 2014;12:7. doi: 10.1186/1478-4505-12-7.

27. Soto-Cáceres V. Calidad del llenado de los certificados de defunción en un hospital público de Chiclayo, Perú 2006. Rev Peru Med Exp Salud Pública. 2008; 25(3):330-32.

28. Valdez W, Gutiérrez C, Siura G. Análisis de la calidad de la certificación de defunciones en la región Ica, 2007. Rev Peru Epidemiol. 2013;17(1).

29. Organización Panamericana de la Salud. El establecimiento de sistemas de información en servicios de atención de salud. Washington D.C: OPS/OMS; 1999.

30. Reyes M, Alarcón J. Registro y flujo de información en el marco de la prevención de la transmisión vertical del VIH. Rev Peru Epidemiol. 2009;13(1).

31. Trujillo OV, Cabezas C, Marín L, Pari J, Salaverry O. Recolección de muestras sanguíneas en poblaciones indígenas. Rev Peru Med Exp Salud Pública. 2012;29(4):575-7.

Correspondencia: Helen Palma Pinedo

Dirección: Los Cóndores 284, Urb. San José, Bellavista, Callao. Perú.

Correo electrónico:helenpalmapinedo@hotmail.com 